\title{
Insights into the molecular biology of epichloë endophyte alkaloid biosynthesis
}

\author{
D.J. FLEETWOOD ${ }^{1}$, B. SCOTT ${ }^{2}$, C.R. VOISEY ${ }^{1}$ and R.D. JOHNSON ${ }^{1}$ \\ ${ }^{1}$ AgResearch Grasslands, Tennent Drive, Palmerston North \\ ${ }^{2}$ Institute of Molecular BioSciences, Massey University, Palmerston North \\ richard.johnson@agresearch.co.nz
}

\begin{abstract}
The production of secondary metabolite alkaloids by epichloë endophytes of grasses is of great interest to agriculture due to the opposing effects of pasture protection against insect herbivory and concomitant toxicity to stock. Recent molecular genetic analysis, that has greatly enhanced our understanding of secondary metabolite biosynthesis, is reviewed and potential outcomes for pastoral agriculture are discussed.
\end{abstract}

Keywords: endophyte, Epichloë, Neotyphodium, alkaloid, secondary metabolite, gene cluster

\section{Introduction}

The interaction between the fungal endophyte Neotyphodium lolii and the forage grass Lolium perenne (perennial ryegrass) is vital to New Zealand agriculture. Alkaloids produced by the endophyte during biotrophic growth can protect host grasses from herbivory from a range of insects, including Argentine stem weevil (Listronotus bonariensis), which would devastate New Zealand ryegrass pastures without the production of alkaloids such as peramine. Despite the net positive effects of endophytes however, some secondary metabolites are toxic to grazing stock, most famously causing the neurotoxicosis ryegrass staggers in livestock by ingestion of endophyte-produced lolitrems.

Strain selection based on chemistry has been successful in identifying $N$. lolii strains (such as AR1) that have low toxicity while retaining resistance to insect herbivory (Tapper \& Latch 1999). However, there are many questions remaining about endophyte secondary metabolism. Compounds such as ergovaline for example, which can cause heat-stress and fescue toxicosis, are also implicated in protection against insects (Ball et al. 1997). Could understanding the biochemical pathways of such compounds lead to identification of non-toxic intermediate compounds that retain insect-protection? Other than the already characterised endophyte-produced alkaloids it is likely that a number of other novel compounds are also produced: could these have positive or negative effects for agriculture? Our aim is to answer these and many other questions using molecular biology - studying secondary metabolism at the gene level. Here we present a review of recent work which has markedly enhanced our understanding of the molecular basis of endophyte secondary metabolite biosynthesis. Possible outcomes for agriculture are discussed.

\section{Secondary metabolite biosynthetic gene clusters - the} LTM gene cluster for indole diterpene biosynthesis

Four alkaloid groups are produced by endophytes with well-characterised effects on the symbiotum. These are peramine, indole diterpenes (such as lolitrem B), lolines and ergot alkaloids (such as ergovaline). Genes for secondary metabolite biosynthesis are often clustered in fungal genomes and genes or gene clusters have been cloned and characterised for each of the four identified endophyte alkaloids.

Indole diterpenes are frequently tremorgenic mycotoxins responsible for neurotoxicoses such as ryegrass staggers (Gallagher et al. 1984). A gene cluster for lolitrem indole diterpene biosynthesis was the first characterised in endophytes and was obtained by first cloning genes for a related indole diterpene compound from the model organism Penicillium paxilli (McMillan et al. 2003; Young et al. 1998, 2001). A geranylgeranyl diphosphate synthase gene from this cluster, $\operatorname{pax} G$, was used as a probe to find the equivalent gene in N. lolii (Young et al. 2005). Chromosome walking (sequencing step by step along the chromosome from a gene of interest), combined with the use of sequences identified in a gene library enriched for endophyte genes expressed in planta, identified further clustered pax gene homologues at a locus labelled LTM proposed to be required for endophyte indole diterpene biosynthesis (Young et al. 2005, 2006).

The biochemical pathway for the synthesis of paxilline has been well characterised in P. paxilli, in which it is the end-point indole diterpene (Saikia et al. 2006, 2007, 2008). Complementation of $P$. paxilli gene deletion mutants with $\mathrm{ltm}$ genes along with analysis of an $\mathrm{ltm} M$ deletion mutant in E. festucae, which does not synthesise any indole diterpenes, supports at least most of the shared genes between the two clusters being responsible for the synthesis of paxilline (Saikia et al. 2008; Young et al. 2005). Genes unique to the epichloë endophyte $L T M$ cluster, $l t m E$, ltmF, $l t m J$ and $\operatorname{ltm} K$, are likely to be responsible for the steps leading to lolitrem B, which are unique to epichloë (Scott et al. 2007).

\section{The perA gene for peramine biosynthesis}

Peramine is a pyrrolopyrazine feeding deterrent to 
Argentine stem weevil (Rowan 1993). Peramine is proposed to be synthesised from arginine and either proline (Rowan 1993) or 1-pyrroline-5-carboxylate (Tanaka et al. 2005). A non-ribosomal peptide synthetase (NRPS) for peramine synthesis, perA, was cloned from $N$. lolii using degenerate polymerase chain reaction (PCR) - a method of amplifying sequence based on conserved enzyme motifs. It is possible that perA is the only gene required for peramine synthesis (Tanaka et al. 2005). Deletion of this gene blocked peramine production, leaving the symbiotum more susceptible to herbivory by Argentine stem weevil (Tanaka et al. 2005). Although production of all alkaloids by endophytes is speculated to confer an ecological advantage to the grass/endophyte symbiota, this study provided rare experimental evidence of an adaptive advantage for production of a secondary metabolite by a fungus.

\section{The $E A S$ gene cluster for ergot alkaloid biosynthesis} Ergot alkaloids include clavines derived from dimethylallyl tryptophan (DMAT), lysergic acid amides and ergopeptines. The main ergopeptine product synthesised by endophytes is ergovaline, which has been implicated in fescue toxicosis, effects of which may include poor weight gain, hyperthermia, convulsions, reduced fertility, gangrene of the extremities and death (Bacon 1995). The gene for the first step in the ergot alkaloid pathway, the DMAT synthase gene $d m a W$, was cloned by degenerate PCR based on the DmaW sequences in the ergot alkaloid-producing pathogenic fungi Claviceps fusiformis and C. purpurea (Wang et al. 2004), similar to the approach taken to identify the ltm genes based on $P$. paxilli sequences. The gene encoding the NRPS LpsA, one of two NRPSs responsible for addition of a tripeptide to lysergic acid to yield lysergyl peptide lactam, the final precursor to ergovaline, was identified by cloning an NRPS from a DNA fragment containing the $d m a W$ gene (Panaccione et al. 2001). Subsequently, one of the NRPS fragments cloned in the perA study was shown to be part of the second NRPS LpsB required for ergovaline synthesis (Fleetwood et al. 2007). Genome walking from this gene identified five other genes clustered with $\operatorname{lps} B$ that were predicted to be required for ergot alkaloid production based on similarity with genes from the C. purpurea ergot alkaloid cluster (Fleetwood et al. 2007).

Deletion of the $\operatorname{lps} B$ gene in E. festucae confirmed its role in the synthesis of ergovaline, causing elimination of ergovaline and lysergic acid amides and accumulation of lysergic acid and other clavine intermediates (Fleetwood et al. 2007). Ergovaline has been implicated in pasture-deterrence of African black beetle (Heteronychus arator) (Ball et al. 1994, 1997). However, insect feeding studies with perennial ryegrass infected with the $\Delta l p s B$ mutant surprisingly showed that ergovaline was not necessary for black beetle feeding deterrence in these symbiota (Fleetwood 2007). Whether clavine intermediate ergot alkaloids or a different alkaloid altogether are responsible for black beetle deterrence in this mutant remains an interesting area of future study.

\section{The $L O L$ gene cluster for loline biosynthesis}

Lolines have potent insecticidal and feeding-deterrent properties and can accumulate up to $2 \%$ of the dry weight of the infected plant's dry mass, far exceeding the biomass of the fungus and the amounts of other alkaloids (Spiering et al. 2002). Until relatively recently, it was not known conclusively if lolines were produced by the plant, the endophyte or both. Blankenship et al. (2001) showed that axenic cultures of $N$. uncinatum, a common meadow fescue endophyte, can produce lolines in minimal media, demonstrating that these products can be solely synthesised by the fungus. However, other strains of endophyte that produce lolines in planta could not be induced to produce lolines in culture (Blankenship et al. 2001). Mendelian analysis of genetic crosses of Loline and Loline- E. festucae showed that loline synthesis segregated as a single genetic locus, designated $L O L$ (Wilkinson et al. 2000). Genes expected to be involved in loline synthesis were subsequently identified in $N$. uncinatum using suppression subtractive hybridisation (Spiering et al. 2002) and genome walking (Spiering et al. 2005). The $l o l$ genes are organised as two almost identical ( $\sim 93 \%$ gene nucleotide identity) gene clusters of nine genes each in the $N$. uncinatum genome, the taxonomic distribution of which strictly correlated with loline production. The role of $l o l C$, a putative pyridoxal phosphate-containing enzyme, was confirmed by RNA interference (RNAi) (Spiering et al. 2005).

\section{Identification of novel secondary metabolite genes}

Non-ribosomal peptide synthetases, such as those involved in peramine and ergovaline biosynthesis, are commonly involved in the synthesis of a variety of secondary metabolites. NRPSs are large multifunctional proteins (Finking \& Marahiel 2004) that synthesise a diverse range of bioactive compounds, some of which serve as pathogenicity factors (Walton 2006; Johnson et al. 2000; Gardiner et al. 2004; Lee et al. 2005), in addition to suggested roles in niche adaptation (Lee et al. 2005). Johnson et al. (2007) sought to survey the numbers of NRPS genes from endophytes of the Neotyphodium/ Epichloë genus in addition to the three NRPS genes involved in the biosynthesis of peramine and ergovaline. A degenerate PCR approach to conserved motifs within NRPS enzymes similar to that used for per $A$ identification was used to identify at least 12 NRPS genes amongst the collective genomes of epichloë species. This is likely to 
be a conservative estimate of the true number of these genes in epichloë endophytes. Based on comparison with characterised genes from other fungi in public databases and the classifications of Lee et al. (2005), the 12 NRPS fragments were predicted to correspond with full-length NRPSs involved in the biosynthesis of siderophores, toxins involved in pathogenesis, $\mathrm{N}$-methylated peptides, and several that could not be classified. This study with NRPSs and a preliminary analysis that has identified at least 10 genes for polyketide synthases, another major class of enzymes involved in secondary metabolite synthesis (C. Voisey, unpublished), suggests that Neotyphodium/Epichloë species are likely to synthesise a much greater number of secondary metabolites than has currently been identified. Some of these may confer additional protection to the host grass against pests and diseases. Gene knockout experiments combined with recently developed unbiased approaches to chemical analysis in endophytes (Cao et al. 2008; Koulman et al. 2007) should enable relatively rapid identification of these novel metabolites.

\section{Impact on the farm - screening and beyond}

The identification of a suite of genes responsible for secondary metabolite production, provides an opportunity to predict the secondary metabolic potential of different endophyte strains by correlating the presence or absence of different genes with known and unknown chemistry. For example, we can already predict the ability of a particular endophyte to synthesise the known toxic or protective alkaloids (Johnson et al. 2007; Tanaka et al. 2005; Young 2005) and in the future it may be possible to correlate unidentified bioactivity with the presence of additional secondary metabolite genes with currently unknown function.

A further benefit of identifying endophyte secondary metabolite genes is the ability to completely dissect biochemical pathways. For example, the analysis of mutants deleted for genes responsible for different steps in the pathway (similar to the $\operatorname{ltm} M, \operatorname{lps} B$ and perA analyses described above) will allow the precise identification of intermediate and end-point compounds with antimammalian and anti-invertebrate properties. This will ultimately enable genetic modification of endophytes to achieve ideal gene sets for maximum pasture protection with minimum animal toxicity, for which no naturally occurring strain has yet been identified.

In conclusion, the molecular dissection of endophyte secondary metabolism is entering an exciting era, with the predicted genes for the synthesis of well-known natural products - indole diterpenes, ergot alkaloids, lolines and peramine - identified. Research focus can now shift to manipulating these known pathways and analysing new predicted secondary metabolite genes with unknown products. These are particularly exciting for future research and may lead to the identification of new bioactivities.

\section{ACKNOWLEDGEMENTS}

We thank Geoff Lane, Brian Tapper, Wade Mace, Karl Fraser and Emily Parker for ongoing chemistry analysis and guidance. This work was supported by FRST grant C10X0203.

\section{REFERENCES}

Bacon, C.W. 1995. Toxic endophyte-infected tall fescue and range grasses: historic perspectives. Journal of Animal Science 73: 861-870.

Ball, O.J-P.; Christensen, M.J.; Prestidge, R.A. 1994. Effect of selected isolates of Acremonium endophytes on adult black beetle (Heteronychus arator) feeding. Proceedings of the 47th New Zealand Plant Protection Conference: 227-231.

Ball, O.J-P.; Miles, C.O.; Prestidge, R.A. 1997. Ergopeptine alkaloids and Neotyphodium loliimediated resistance in perennial ryegrass against adult Heteronychus arator (Coleoptera: Scarabaeidae). Journal of Economic Entomology 90: 1382-1391.

Blankenship, J.D.; Spiering, M.J.; Wilkinson, H.H.; Fannin, F.F.; Bush, L.P.; Schardl, C.L. 2001. Production of loline alkaloids by the grass endophyte, Neotyphodium uncinatum, in defined media. Phytochemistry 58: 395-401.

Cao, M.; Koulman, A.; Johnson, L.J.; Lane, G.A.; Rasmussen, S. 2008. Advanced data-mining strategies for the analysis of direct-infusion ion trap mass spectrometry data from the association of perennial ryegrass with its endophytic fungus, Neotyphodium lolii. Plant Physiology 146: 1501-1514.

Fleetwood, D.J. 2007. Molecular characterisation of the $E A S$ gene cluster for ergot alkaloid biosynthesis in epichloë endophytes of grasses. PhD thesis, Massey University, Palmerston North.

Fleetwood, D.J.; Scott, B.; Lane, G.A.; Tanaka, A.; Johnson, R.D. 2007. A complex ergovaline gene cluster in epichloë endophytes of grasses. Applied and Environmental Microbiology 73: 2571-2579.

Gallagher, R.T.; Hawkes, A.D.; Steyn, P.S.; Vleggaar, R. 1984. Tremorgenic neurotoxins from perennial ryegrass causing ryegrass staggers disorder of livestock: Structure elucidation of lolitrem B. Journal of the Chemical Society, Chemical Communications: 614-616.

Johnson, R.; Voisey, C.; Johnson, L.; Pratt, J.; Fleetwood, D.; Khan, A.; Bryan, G. 2007. Distribution of NRPS gene families within the Neotyphodium/Epichloë complex. Fungal Genetics and Biology 44: 1180-1190. Keller, N.P.; Hohn, T.M. 1997. Metabolic pathway gene 
clusters in filamentous fungi. Fungal Genetics and Biology 21: 17-29.

Koulman, A.; Tapper, B.A.; Fraser, K.; Cao, M.; Lane, G.A.; Rasmussen, S. 2007. High-throughput directinfusion ion trap mass spectrometry: a new method for metabolomics. Rapid Communications in Mass Spectrometry 21: 421-428.

Kutil, B.L.; Greenwald, C.; Liu, G.; Spiering, M.J.; Schardl, C.L.; Wilkinson, H.H. 2007. Comparison of loline alkaloid gene clusters across fungal endophytes: Predicting the co-regulatory sequence motifs and the evolutionary history. Fungal Genetics and Biology 44:1002-1010.

May, K.J.; Bryant, M.K.; Zhang, X.; Ambrose, B.; Scott, B. 2008. Patterns of expression of a lolitrem biosynthetic gene in the Epichloe festucae-perennial ryegrass symbiosis. Molecular Plant-Microbe Interactions 21: 188-197.

McMillan, L.K.; Carr, R.L.; Young, C.A.; Astin, J.W.; Lowe, R.G.; Parker, E.J.; Jameson, G.B.; Finch, S.C.; Miles, C.O.; McManus, O.B.; Schmalhofer, W.A.; Garcia, M.L.; Kaczorowski, G.J.; Goetz, M.; Tkacz, J.S.; Scott, B. 2003. Molecular analysis of two cytochrome P450 monooxygenase genes required for paxilline biosynthesis in Penicillium paxilli, and effects of paxilline intermediates on mammalian maxi-K ion channels. Molecular Genetics and Genomics 270: 9-23.

Panaccione, D.G.; Wang, J.; Young, C.A.; Schardl, C.L.; Scott, B.; Damrongkool, P. 2001. Elimination of ergovaline from a grass-Neotyphodium endophyte symbiosis by genetic modification of the endophyte. Proceedings of the National Academy of Sciences of the United States of America 98: 12820-12825.

Rowan, D.D. 1993. Lolitrems, peramine and paxilline: mycotoxins of the ryegrass/endophyte interaction. Agriculture Ecosystems and Environment 44: 1-4.

Saikia, S.; Parker, E.J.; Koulman, A.; Scott, B. 2006. Four gene products are required for the fungal synthesis of the indole-diterpene, paspaline. FEBS Letters 580: 16251630.

Saikia, S.; Parker, E.J.; Koulman, A.; Scott, B. 2007. Defining paxilline biosynthesis in Penicillium paxilli: functional characterisation of two cytochrome P450 monooxygenases. Journal of Biological Chemistry 282: 16829-16837.

Saikia, S.; Nicholson, M.J.; Young, C.; Parker, E.J.; Scott, B. 2008. The genetic basis for indole-diterpene chemical diversity in filamentous fungi. Mycological Research 112: 184-199.

Scott, B.; Takemoto, D.; Tanaka, A.; Young, C.A.; Bryant, M.K.; May, K.J. 2007. Functional analysis of the Epichloe festucae-perennial ryegrass symbiosis. Proceedings of the 6th International Symposium on Fungal Endophytes of Grasses. Grassland Research and Practice Series
13: 433-441.

Spiering, M.J.; Wilkinson, H.H.; Blankenship, J.D.; Schardl, C.L. 2002. Expressed sequence tags and genes associated with loline alkaloid expression by the fungal endophyte Neotyphodium uncinatum. Fungal Genetics and Biology 36: 242-254.

Spiering, M.J.; Moon, C.D.; Wilkinson, H.H.; Schardl, C.L. 2005. Gene clusters for insecticidal loline alkaloids in the grass-endophytic fungus Neotyphodium uncinatum. Genetics 169: 1403-1414.

Tanaka, A.; Tapper, B.A.; Popay, A.; Parker, E.J.; Scott, B. 2005. A symbiosis expressed non-ribosomal peptide synthetase from a mutualistic fungal endophyte of perennial ryegrass confers protection to the symbiotum from insect herbivory. Molecular Microbiology 57: 10361050.

Tapper, B.A.; Latch, G.C.M. 1999. Selection against toxin production in endophyte-infected perennial ryegrass. Ryegrass Endophyte: An Essential New Zealand Symbiosis. Grassland Research and Practice 7: 107111.

Wang, J.; Machado, C.; Panaccione, D.G.; Tsai, H.F.; Schardl, C.L. 2004. The determinant step in ergot alkaloid biosynthesis by an endophyte of perennial ryegrass. Fungal Genetics and Biology 41: 189-198.

Wilkinson, H.H.; Siegel, M.R.; Blankenship, J.D.; Mallory, A.C.; Bush, L.P.; Schardl, C.L. 2000. Contribution of fungal loline alkaloids to protection from aphids in a grass-endophyte mutualism. Molecular Plant-Microbe Interactions 13: 1027-1033.

Young, C.A. 2005. The indole diterpene gene cluster from the ryegrass endophyte, Neotyphodium lolii, is required for the biosynthesis of lolitrem B, a bioprotectve alkaloid. $\mathrm{PhD}$ thesis, Massey University, Palmerston North.

Young, C.; Itoh, Y.; Johnson, R.; Garthwaite, I.; Miles Christopher, O.; Munday-Finch Sarah, C.; Scott, B. 1998. Paxilline-negative mutants of Penicillium paxilli generated by heterologous and homologous plasmid integration. Current Genetics 33:368-377.

Young, C.A.; McMillan, L.; Telfer, E.; Scott, B. 2001. Molecular cloning and genetic analysis of an indolediterpene gene cluster from Penicillium paxilli. Molecular Microbiology 39: 754-764.

Young, C.A.; Bryant, M.K.; Christensen, M.J.; Tapper, B.A.; Bryan, G.T.; Scott, B. 2005. Molecular cloning and genetic analysis of a symbiosis-expressed gene cluster for lolitrem biosynthesis from a mutualistic endophyte of perennial ryegrass. Molecular Genetics and Genomics 274: 13-29.

Young, C.A.; Felitti, S.; Shields, K.; Spangenberg, G.; Johnson, R.D.; Bryan, G.T.; Saikia, S.; Scott, B. 2006. A complex gene cluster for indole-diterpene biosynthesis in the grass endophyte Neotyphodium lolii. Fungal Genetics and Biology 43: 679-693. 